02

\title{
Фотофизические процессы в молекулах галогенпроизводных флуоресцеина в анионных обратных мицеллах
}

\author{
() О.И. Волкова ${ }^{1}$, А.А. Кулешова ${ }^{1}$, Б.Н. Корватовский ${ }^{2}$, А.М. Салецкий ${ }^{1}$ \\ ${ }^{1}$ Физический фракультет МГУ им. М.В. Ломоносова, \\ 119991 Москва, Россия \\ ${ }^{2}$ Биологический фракультет МгУ им. М.В. Ломоносова, \\ 119234 Москва, Россия \\ I e-mail:sam@physics.msu.ru
}

Поступила в редакцию 24.06.2020 г.

В окончательной редакции 31.08.2020 г.

Принята к публикации 04.09.2020 г.

Исследованы фотофизические процессы в водно-мицеллярных растворах галогенпроизводных флуоресцеина: эозина (Э), эритрозина (ЭР) и бенгальского розового (БР), методами динамического рассеяния света, стационарной и времяразрешенной флуоресцентной спектроскопии. Установлено, что внедрение молекул красителей в обратные мицеллы АОТ вызывает увеличение их гидродинамических радиусов $R_{\mathrm{h}}$. Изучена кинетика флуоресценции исследованных молекул красителей в обратных мицеллах. Установлено уменьшение среднего времени возбужденного состояния $\langle\tau\rangle$ с ростом $R_{\mathrm{h}}$ для Э, ЭР и БР, которое связано с ростом подвижности молекул воды и ослаблением эффекта геометрического ограничения молекул красителей. Измерены степени анизотропии флуоресценции $r$ молекул красителей в обратных мицеллах, показано, что в мицеллярных системах $r$ больше, чем в водных растворах и уменьшаются с ростом $R_{\mathrm{h}}$. Для исследованных молекул красителей в мицеллярных системах определено время вращательной корреляции $\theta$, которое уменьшается для всех исследованных красителей с ростом $R_{\mathrm{h}}$, что указывает на уменьшение микровязкости ограниченной водной среды внутри мицеллы. При этом $\theta_{\mathrm{E}}>\theta_{\mathrm{ER}}>\theta_{\mathrm{BR}}$, т. е. в значении времени вращательной корреляции проявляется „Эффект внутреннего тяжелого атома“.

Ключевые слова: красители, галогенпроизводные флуоресцеина, обратные мицеллы, динамическое рассеяние света, спектры, анизотропия флуоресценции, среднее время жизни возбужденного состояния, время вращательной корреляции.

DOI: $10.21883 /$ OS.2020.12.50319.181-20

\section{Введение}

В последние годы спектрально-люминесцентные методы широко применяются для мониторинга состояния клеток, белков, живых тканей и других биологических объектов. Большую роль в таких исследованиях играет метод флуоресцентного зонда, в качестве которого используются молекулы красителей. Исследования взаимодействия белок-краситель позволяют определять структурные изменения в среде, окружающей белок. Большинство исследований in vitro посвящено изучению белков в сильно разбавленных буферных растворах, в то время как биомолекулы функционируют в замкнутом пространстве биологической клетки (в замкнутом наноокружении). Поэтому для исследований, приближенных к реальным условиям, необходимо установление влияния пространственного ограничения на спектральнолюминесцентные характеристики как белков, так и красителей-зондов. Такое пространственное ограничение возможно осуществить в четырех различных модельных структурах: нанопорах (представляют собой твердые тела), обратных мицеллах, органических растворителях и гидратных оболочках (в частности, гидратных оболочках белков).
К первому типу модельных структур относится пористое стекло, которое имеет большое отношение площади поверхности пор к их объему. Большой свободный объем в таких структурах играет существенную роль в процессах трансляционной и вращательной диффузии адсорбированных в порах молекул, поэтому пористое стекло используется для исследования поведения молекул воды [1] и сложных молекул, обладающих люминесцентной способностью [2,3]. При этом существенное влияние на фотофизические процессы в таких системах играют поверхности пор, поэтому проявляется интерес к изменению свойств поверхностей путем их покрытия различными веществами. Наиболее перспективным является использования для этих целей наноструктуры с „мягкими“ стенками, которые могут быть реализованы в микроэмульсиях.

К таким структурам относятся обратные мицеллы, представляющие собой наноразмерные частицы, состоящие из молекул поверхностно-активного вещества, которые собираются вокруг водного ядра, суспендированного в неполярном растворителе. Размер мицелл можно варьировать, изменяя соотношение воды и поверхностноактивного вещества - так, размер обратных мицелл натрий 1,4-бис (2-этилгексил) сульфосукцинат (АОТ) 
определяется степенью гидратации $w=\frac{\left[\mathrm{H}_{2} \mathrm{O}\right]}{[\mathrm{AOT}]}$, где [АОТ] и $\left[\mathrm{H}_{2} \mathrm{O}\right]-$ молярные концентрации каждого вида в растворе. Наноразмерное водное ядро обратных мицелл действует как „нанореактор“, внутри которого контролируемые реакции приводят к образованию широкого спектра наноструктур с относительно узким распределением по размерам [4]. Эти системы привлекли большое внимание, поскольку они охватывают впечатляюще широкий спектр практических применений, наибольший прогресс при этом был достигнут в синтезе наночастиц [5-7] и в разработке передовых средств доставки лекарств $[8,9]$.

В последние несколько лет появилось большое количество работ, посвященных исследованию молекул красителей в анионных обратных мицеллах АОТ методами линейной [10-12] и нелинейной $[13,14]$ спектроскопии. При этом исследования в системе обратная мицелламолекула красителя обычно проводятся с использованием катионных красителей. Очень редко используются анионные красители. К небольшому числу работ, посвященных изучению систем обратная мицелла-анионный краситель, можно отнести [15-17].

В данной статье представлены результаты исследования фотофизических процессов в молекулах галогенпроизводных флуоресцеина: эозина (Э), эритрозина (ЭР), бенгальского розового (БР) (анионных при физиологических значениях $\mathrm{pH}$ 7.4) в обратных мицеллах, проведенного методами динамического рассеяния света, стационарной и времяразрешенной флуоресценции. Выбор этих красителей обусловлен тремя причинами. Вопервых, молекулы красителей флуоресцеинового ряда широко применяются в медицине как в качестве контрастных веществ, так и в фотодинамической терапии, в частности, для лечения меланомы [18]. Имея высокие квантовые выходы генерации синглетного кислорода, эти красители становятся потенциальными фотосенсибилизаторами для нового направления фотодинамической терапии - антимикробной фотодинамической терапии [19-21]. Во-вторых, представленный ряд флуорофоровых красителей характеризуется последовательным замещением атомов водорода атомами галогенов (бром, йод и хлор), которое позволяет плавно варьировать их физико-химическими свойствами перераспределением зарядов между атомами, изменением электронных и пространственных структур и др. В-третьих, эти красители широко используются для исследования биологических объектов, в частности белков, методами спектроскопии комбинационного рассеяния света [22] и флуоресцентной спектроскопии [23,24].

\section{Эксперимент}

\section{Материалы}

В работе использовали sodium 1,4-bis (2-ethylhexyl) sulfosuccinate AOT (Sigma Aldrich, Германия, чистота $>99 \%$ ), декан (Sigma Aldrich, Германия, чистота $>99 \%$ ) и анионные красители эозин (Э), эритро-<smiles>[X]c1cc2c(-c3c([Y])c([Y])c([Y])c([Y])c3C(=O)O)c3cc([X])c(=O)c([X])c-3oc2c([X])c1O</smiles>

Рис. 1. Химическая формула исследованных красителей: Э $(\mathrm{X}=\mathrm{Br}, \mathrm{Y}=\mathrm{H})$, ЭP $(\mathrm{X}=\mathrm{I}, \mathrm{Y}=\mathrm{H})$, БP $(\mathrm{X}=\mathrm{I}, \mathrm{Y}=\mathrm{Cl})$.

зин (ЭР) и бенгальский розовый (БР) (рис. 1) фирмы Sigma Aldrich без дополнительной очистки. Все растворы приготавливались из сухих реактивов АОТ, красителя и гептана. Сначала создавался $10 \%$ по массе раствор АОТ в гептане: взвешивались $1 \mathrm{~g}$ АОТ и $9 \mathrm{~g}$ гептана. Затем к $2 \mathrm{ml}$ АОТ в гептане добавлялись красители, разведенные в воде в количестве $100-500^{-5} \mathrm{~mol} / 1$ (число гидратации $w$ варьировалось от 0 до 70) с концентрацией $C=10^{-5} \mathrm{~mol} / \mathrm{l}$.

\section{Определение размеров мицелл}

Для определения размеров мицелл использовался метод динамического рассеяния света. Методика определения $R_{\mathrm{h}}$ для мицеллярных систем подробно описана в [25] и основана на определении временной корреляционной функции рассеянного света в растворах:

$$
G(\tau)=\frac{\langle I(t) I(t+\tau)\rangle}{\left\langle I^{2}(t)\right\rangle},
$$

где $I(t)$ и $I(t+\tau)$ - интенсивность рассеянного света в моменты $t$ и $t+\tau$ соответственно.

Для монодисперсного коллоидного раствора

$$
G(\tau)=A \exp (-\Gamma \tau),
$$

где $A-$ постоянная прибора, $\Gamma$ - скорость релаксации, связанная с коэффициентом диффузии $D$ следующим соотношением:

$$
\Gamma=\frac{1}{\tau_{K}}=D q^{2},
$$

$q-$ вектор рассеяния, модуль которого равен

$$
q=4 \pi \frac{n}{\lambda} \sin \left(\frac{\varphi}{2}\right),
$$

где $\varphi-$ угол рассеяния (в наших экспериментах $\left.\varphi=90^{\circ}\right), \lambda$ - длина волны рассеянного света, $n-$ показатель преломления раствора. Таким образом, определив Г из $G(\tau)$, можно определить коэффициент диффузии $D$. 
Зная коэффициент диффузии, в рамках модели СтоксаЭйштейна-Дебая в предположении сферической формы мицелл определялся их гидродинамический радиус:

$$
R_{\mathrm{h}}=\frac{k T}{6 \pi \eta D}
$$

где $k-$ постоянная Больцмана, $T-$ температура, $\eta-$ вязкость растворителя.

Измерение динамического рассеяния света и анализ размеров частиц осуществлялись на приборе Photocor Compact (использовался полупроводниковый лазер с $\lambda=638 \mathrm{~nm}$, мощность $25 \mathrm{~mW}$ ). Погрешность измерения гидродинамического радиуса мицелл $0.3 \mathrm{~nm}$.

\section{Измерение спектрально-люминесцентных характеристик растворов}

Регистрация спектров поглощения водно-мицеллярных растворов красителей была проведена на спектрофотометре Perkin Elmer Lambda 35 (спектральная ширина щели $0.5 \mathrm{~nm}$ ). Регистрация спектров флуоресценции молекул красителей в мицеллярных растворах проводилась на спектрофлуориметре Perkin Elmer LS 55 (спектральное разрешение $0.1 \mathrm{~nm}$, воспроизводимость длин волн $0.5 \mathrm{~nm}$ ).

\section{Степень анизотропии флуоресценции}

Степень анизотропии флуоресценции $r$ определялась по формуле

$$
r=\frac{I_{\mathrm{VV}}-k I_{\mathrm{VH}}}{I_{\mathrm{VV}}+2 k I_{\mathrm{VH}}}
$$

где $I_{\mathrm{VV}}$ - интенсивность флуоресценции при вертикальной ориентации поляризаторов возбуждения и излучения; $I_{\mathrm{VH}}-$ интенсивность флуоресценции, полученная при вертикальной ориентации поляризатора возбуждения и горизонтальной ориентации поляризатора излучения; $k=\frac{I_{\mathrm{HV}}}{I_{\mathrm{HH}}} ; I_{\mathrm{HH}}-$ интенсивность флуоресценции при горизонтальной ориентации поляризаторов возбуждения и излучения; $I_{\mathrm{HV}}$ - интенсивность флуоресценции, полученная при горизонтальной ориентации поляризатора возбуждения и вертикальной ориентации поляризатора излучения.

\section{Исследование времяразрешенной флуоресценции}

Измерение времени жизни флуоресценции красителей проводилось с использованием прибора Simple Tau 140, работающего в режиме счета фотонов (Becker \& Hickl, Германия). Флуоресценцию красителей возбуждали лазером BDS-SM510 (длина волны $510 \mathrm{~nm}$, длительность импульса $40 \mathrm{ps}$ ). Флуоресценция регистрировалась 16-канальным фотоприемником K5900 (Hamamatsu, Япония), перед которым находился решетчатый полихроматор (600lines/mm). Ширина спектра на выходе полихроматора составляла $200 \mathrm{~nm}$, что соответствовало
$12.5 \mathrm{~nm}$ на канал. Время интегрирования составляло $30 \mathrm{~s}$. Кинетические кривые флуоресценции были обработаны с использованием программы SPCImage (Becker \& Hickl, Германия). Погрешность определения времени жизни возбужденного состояния молекул красителей $0.05 \mathrm{~ns}$.

Кривая затухания флуоресценции аппроксимировалась несколькими экспонентами:

$$
F(t)=\sum_{i=1}^{N} A_{i} e^{\left(-\frac{t}{\tau_{i}}\right)},
$$

где $N$ - число экспонент, $t-$ время, $\tau_{i}-$ время жизни флуоресценции, $A_{i}$ - амплитуда.

Все измерения были проведены при температуре $295 \mathrm{~K}$.

\section{Результаты и их обсуждения}

Методом динамического рассеяния света были определены гидродинамические радиусы исследованных мицелл. В табл. 1 представлены $R_{\mathrm{h}}$ для четырех значений степени гидратации $w$, определенные с помощью соотношения (4) для мицелл с внедренными молекулами красителей и без них. Из табл. 1 видно, что гидродинамический радиус мицелл с внедренными молекулами красителя больше, чем для водных мицелл для всех исследованных значений $w$. В табл. 1 также представлены значения $\Delta R_{\mathrm{h}}=R_{\mathrm{h}}^{\text {Dуе }}-R_{\mathrm{h}}$ (здесь $\underset{\mathrm{h}}{\mathrm{Dye}}-$ гидродинамический радиус мицелл, содержащих молекулы красителей, $R_{\mathrm{h}}$ - гидродинамический радиус мицелл, не содержащих молекулы красителей) для исследованных значений $w$. Видно, что для всех трех красителей наблюдается увеличение разности $\Delta R_{\mathrm{h}}$. Для БР $\Delta R_{\mathrm{h}}$ увеличивается незначительно и практически не зависит от $\omega, \Delta R_{\mathrm{h}}$ для Э и ЭР изменяется значительно. Наибольшее изменение $\Delta R_{\mathrm{h}}$ наблюдается для ЭР, меньшее - для Э, но при этом наблюдается увеличение $\Delta R_{\mathrm{h}}$ с ростом $w$. Это изменение $\Delta R_{\mathrm{h}}$ связано с тем, что при увеличении гидродинамического радиуса мицеллы происходит изменение свойств структурированной воды, сопровождающееся изменением электростатического взаимодействия между анионами красителей и гидратированными группами поверхностно-активных веществ (ПАВ). В дальнейшем для описания люминесцентных характеристик красителей в водно-мицеллярных растворах использовались значения $R_{\mathrm{h}}$ для мицелл с внедренными молекулами красителей.

Были зарегистрированы спектры поглощения и флуоресценции красителей в мицеллярных растворах при различных значениях их гидродинамического радиуса $R_{\mathrm{h}}$. В качестве примера на рис. 2 представлены спектры поглощения, а на рис. 3 - спектры флуоресценции исследованных красителей в водно-мицеллярных растворах для гидродинамического радиуса $R_{h}=4 \mathrm{~nm}$. Из рис. 2 и 3 видно, что длины волн максимумов поглощения и флуоресценции для исследованных красителей отличаются. Так, максимумы поглощения наблюдаются 
Таблица 1. Значения гидродинамического радиуса мицелл $R_{\mathrm{h}}$ и $\Delta R_{\mathrm{h}}$ для исследованных систем (значения $\Delta R_{\mathrm{h}}$ указаны в скобках)

\begin{tabular}{c|c|c|c|c}
\hline \multirow{2}{*}{$w$} & \multicolumn{4}{|c}{$R_{\mathrm{h}, \mathrm{nm}}$} \\
\cline { 2 - 5 } & Вода & $Э$ & ЭР & БР \\
\hline 18.6 & 3.3 & $4(0.7)$ & $4.8(1.5)$ & $4.6(1.3)$ \\
37 & 6.8 & $7.4(0.6)$ & $10.5(3.7)$ & $7.9(1.1)$ \\
55 & 11 & $14.8(3.8)$ & $15.8(4.8)$ & $12.5(1.5)$ \\
70 & 15 & $18.6(3.6)$ & $20(5)$ & $17(2)$
\end{tabular}

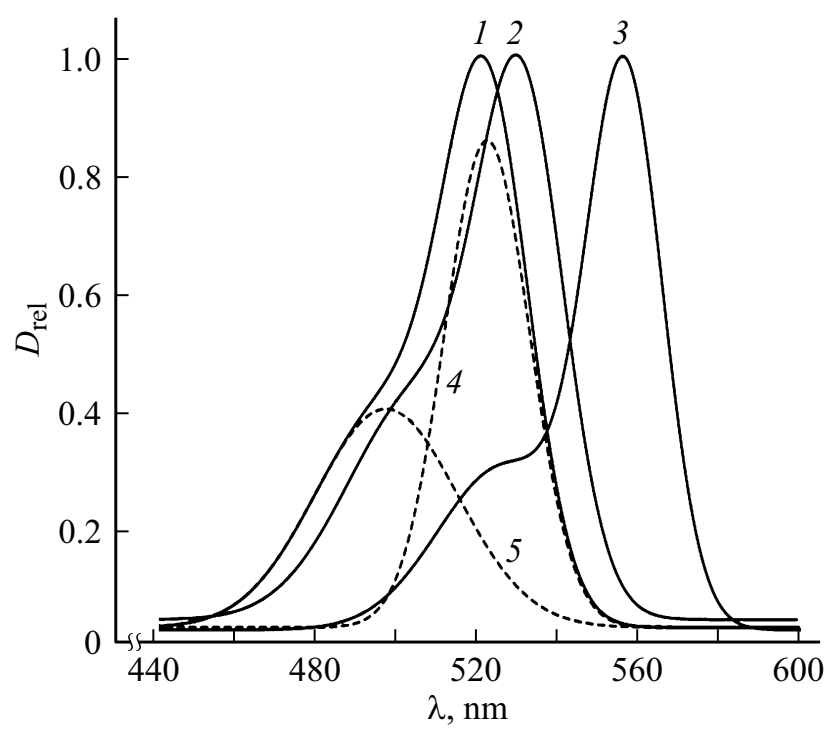

Рис. 2. Нормированные спектры поглощения Э (1), ЭР (2) и БР (3) в водно-мицеллярных растворах с $R_{\mathrm{h}}=4 \mathrm{~nm}$. Кривые 4,5 - гауссовы компоненты спектра поглощения эозина. Концентрация красителя равна $10^{-5} \mathrm{~mol} / \mathrm{l}$.

при 520 (Э), 531 (ЭР), 556 nm (БР) (рис. 2). Спектры флуоресценции этих красителей имеют максимумы при 546 (Э), 550 (ЭР), $578 \mathrm{~nm}$ (БР) (рис. 3).

Спектры поглощения красителей имеют две полосы: длинноволновую (с длиной максимума $\lambda_{1}$ ) и коротковолновую (с длиной волны максимума $\lambda_{2}$ ), Полоса с $\lambda_{1}$ относится к дианионной форме красителей, а $\lambda_{2}-$ к их анионной форме [26]. Спектры поглощения были разделены на две гауссовы полосы. На рис. 2 в качестве примера представлены две выделенные компоненты для Э: полоса поглощения дианионной формы (кривая 4) и полоса поглощения анионной формы (кривая 5).

Эти полосы были выделены для всех красителей и всех значений $R_{\mathrm{h}}$, а затем определены их интенсивности. На рис. 4 представлены зависимости отношения $\frac{I_{\lambda_{2}}}{I_{\lambda_{1}}}$ $\left(I_{\lambda_{1}}\right.$ - интенсивность дианионной полосы, $I_{\lambda_{2}}-$ интенсивность анионной полосы) от гидродинамического радиуса мицелл $R_{\mathrm{h}}$ для всех красителей. Из рис. 4 видно, что с ростом $R_{\mathrm{h}}$ наблюдается увеличение отношения $\frac{I_{\lambda_{2}}}{I_{\lambda_{1}}}$. Результаты исследования спектральных характеристик красителей, представленные на рис. 4, указывают на то, что с ростом $R_{\mathrm{h}}$ увеличивается вклад анионной формы красителя в формирование спектров поглощения. В связи с тем, что интенсивность поглощения пропорциональна концентрации молекул, представленные на рис. 4 данные указывают на то, что с ростом $R_{\mathrm{h}}$ происходит изменение соотношения концентраций дианионной и анионной форм красителей. Это должно сказаться на флуоресцентных характеристиках исследованных растворов - в частности, на среднем времени флуоресценции и степени анизотропии свечения.

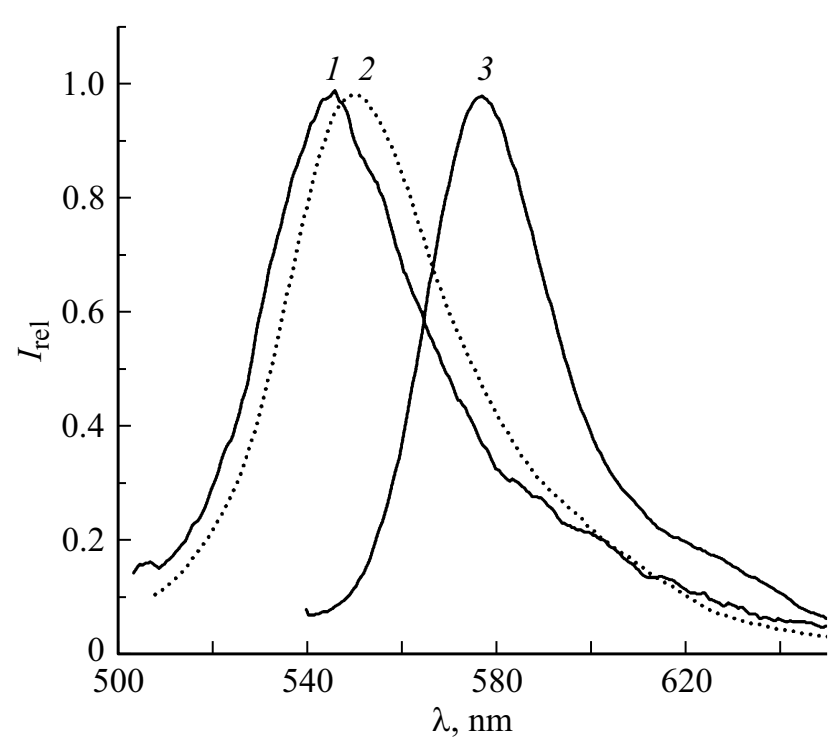

Рис. 3. Нормированные спектры флуоресценции Э (1), ЭР (2) и БР (3) в водно-мицеллярных растворах с $R_{\mathrm{h}}=4 \mathrm{~nm}$. Концентрация красителя равна $10^{-5} \mathrm{~mol} / \mathrm{l}$.

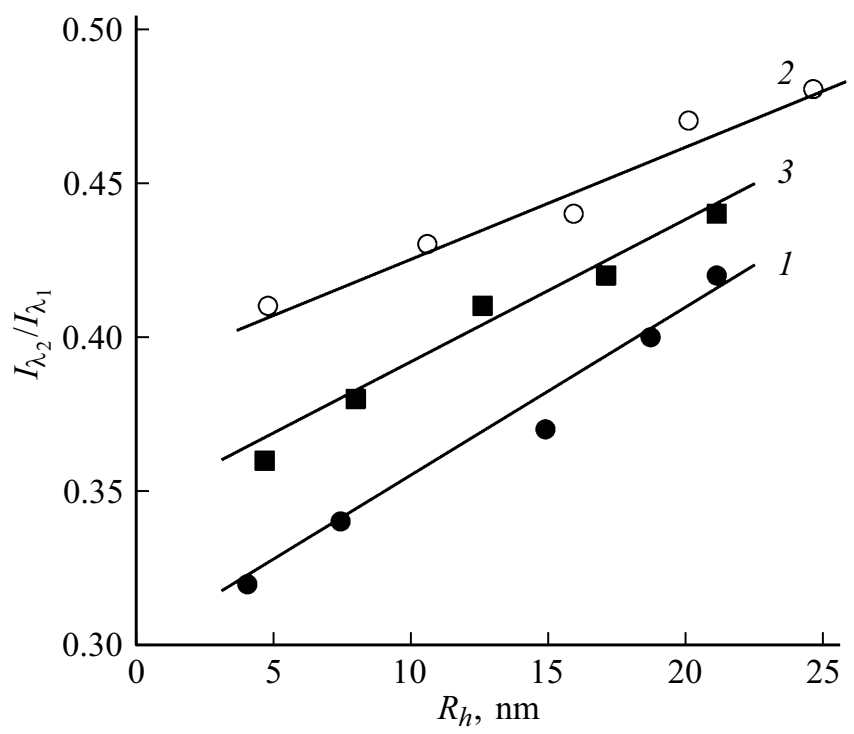

Рис. 4. Зависимости отношения интенсивностей поглощения анионной и дианионной полос $\frac{I_{\lambda_{2}}}{I_{\lambda_{1}}}$ от гидродинамического радиуса $R_{\mathrm{h}}$ для Э (1), ЭР (2) и БР (3). 
Таблица 2. Параметры $A_{1}, \tau_{1}, A_{2}$ и $\tau_{2}$ для различных красителей и значений $R_{\mathrm{h}}$

\begin{tabular}{|c|c|c|c|c|c|}
\hline Краситель & $R_{\mathrm{h}}, \mathrm{nm}$ & $A_{1}, \%$ & $\tau_{1}, \mathrm{~ns}$ & $A_{2}, \%$ & $\tau_{2}, \mathrm{~ns}$ \\
\hline \multirow[t]{5}{*}{$Э$} & 3.7 & 95.0 & 1.92 & 5.0 & 3.84 \\
\hline & 7.3 & 99.0 & 1.84 & 1.0 & 3.62 \\
\hline & 12.0 & 95.0 & 1.6 & 5.0 & 1.72 \\
\hline & 17.5 & 97.0 & 1.39 & 3.0 & 5.25 \\
\hline & \multicolumn{4}{|c|}{ Водный раствор } & 1.21 \\
\hline \multirow[t]{5}{*}{ ЭР } & 4.8 & 98.0 & 0.38 & 2.0 & 1.21 \\
\hline & 10.5 & 95.0 & 0.33 & 5.0 & 1.24 \\
\hline & 15.8 & 96.0 & 0.3 & 4.0 & 1.63 \\
\hline & 20.0 & 99.0 & 0.32 & 1.0 & 3.82 \\
\hline & \multicolumn{4}{|c|}{ Водный раствор } & 2.13 \\
\hline \multirow[t]{5}{*}{ БР } & 4.6 & 94.0 & 0.45 & 6.0 & 3.48 \\
\hline & 7.9 & 96.0 & 0.51 & 4.0 & 3.9 \\
\hline & 12.5 & 96.0 & 0.47 & 4.0 & 3.22 \\
\hline & 17.0 & 91.0 & 0.24 & 9.0 & 2.94 \\
\hline & \multicolumn{4}{|c|}{ Водный раствор } & 2.94 \\
\hline
\end{tabular}

Ниже приведены результаты измерения времени затухания флуоресценции исследованных систем. Удовлетворительное совпадение с экспериментальными кривыми дает аппроксимация затухания флуоресценции красителей в мицеллярных растворах двумя экспонентами:

$$
F(t)=A_{1} e^{\left(-t / \tau_{1}\right)}+A_{2} e^{\left(-t / \tau_{2}\right)} .
$$

Здесь $A_{i}$ - амплитуды экспонент, $\tau_{i}-$ время затухания экспонент, $t$ - время после возбуждения флуоресценции. В табл. 2 представлены значения $A_{1}, \tau_{1}, A_{2}$ и $\tau_{2}$ для растворов красителей в мицеллах для различных значений $R_{\mathrm{h}}$. В этой же таблице представлены значения времен жизни возбужденного состояния молекул красителей в водных растворах. Для водных растворов красителей наблюдается моноэкспоненциальное затухание свечения, в то время как для мицеллярных растворов затухание свечения описывается двумя экспонентами. Наличие двухэкспоненциальных зависимостей затухания свечения указывает на то, что внутри обратной мицеллы молекулы красителей находятся в различном окружении.

Для анализа влияния степени гидратации на кинетические характеристики флуоресценции молекул красителей удобно использовать среднее время жизни флуоресценции:

$$
\langle\tau\rangle=\frac{A_{1} \tau_{1}+A_{2} \tau_{2}}{A_{1}+A_{2}} .
$$

На рис. 5 представлены зависимости $\langle\tau\rangle$ от $R_{\mathrm{h}}$ для исследованных систем. В ряду красителей Э-ЭР-БР происходит увеличение энергии спин-орбитальнего взаимодействия за счет эффекта внутреннего тяжелого атома. Замещающие тяжелые атомы усиливают спинорбитальное взаимодействие в молекулах красителей, что приводит к уменьшению $\langle\tau\rangle$. При этом с ростом $R_{\mathrm{h}}$ время $\langle\tau\rangle$ уменьшается (рис. 5). Эти результаты

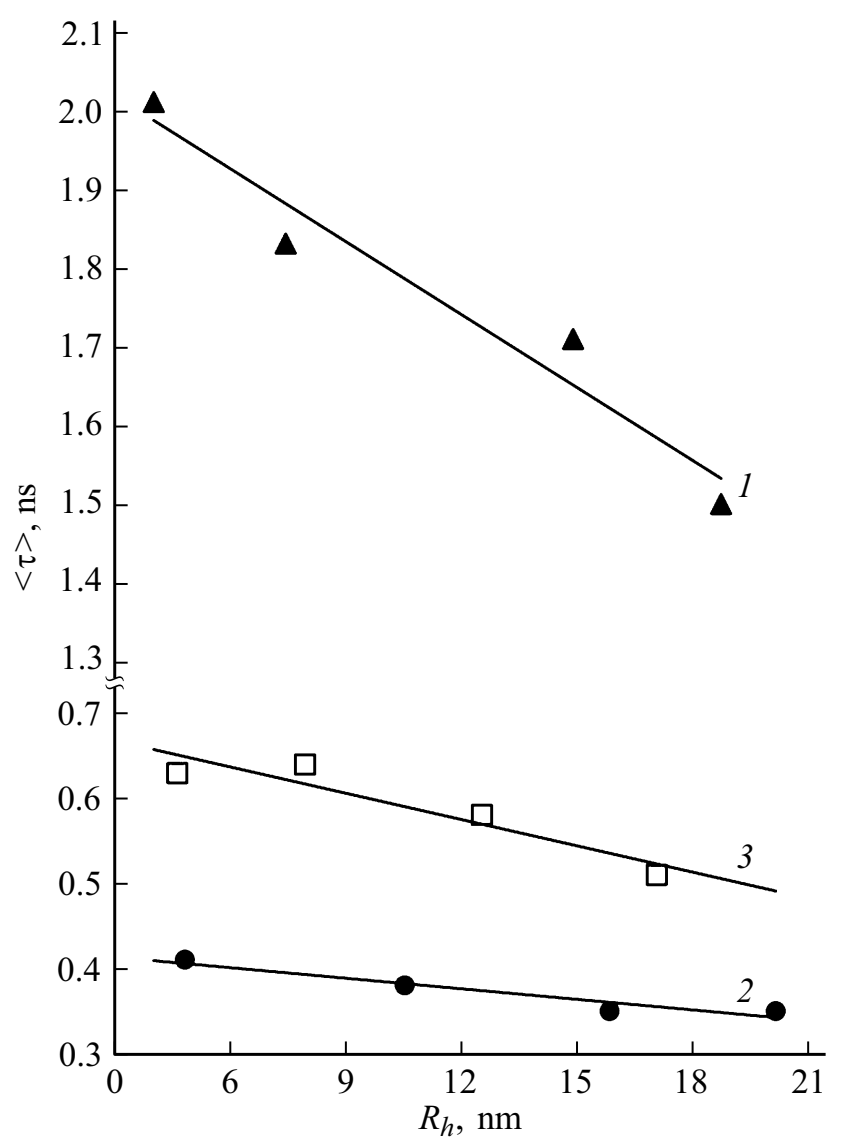

Рис. 5. Зависимости среднего времени жизни флуоресценции $\langle\tau\rangle$ от гидродинамического радиуса мицелл $R_{\mathrm{h}}$ для Э $(1)$, ЭР (2) и БР (3).

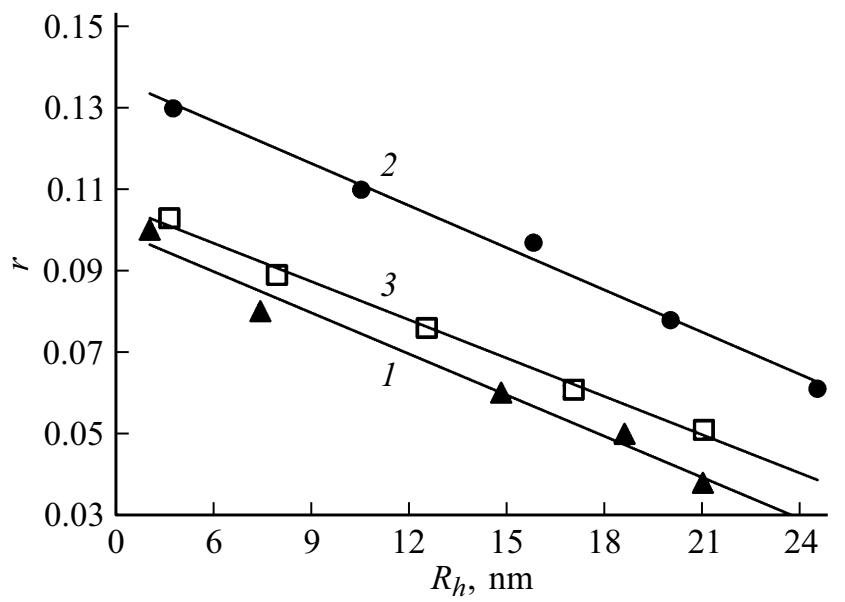

Рис. 6. Зависимости степени анизотропии флуоресценции $r$ от гидродинамического радиуса $R_{\mathrm{h}}$ для Э $(1)$, ЭР (2) и БР (3).

указывают на то, что с ростом $R_{\mathrm{h}}$ ограничение движения, испытываемое флуорофором при связывании с обратными мицеллами, уменьшается.

Таким образом, увеличение размера обратных мицелл приводит к ослаблению эффекта геометрического ограничения молекул исследованных красителей, что 
должно сказываться на значениях степени анизотропии флуоресценции. Были определены значения степени анизотропии флуоресценции $r$ для молекул красителей в обратных мицеллах с различными $R_{\mathrm{h}}$. Степень анизотропии флуоресценции флуорофора зависит от вязкости окружения, а также от времени жизни возбужденного состояния молекул красителей. На рис. 6 представлены зависимости $r\left(R_{\mathrm{h}}\right)$ для исследованных красителей. Из рис. 6 видно, что для красителей с коротким временем жизни возбужденного состояния (ЭР и БР) $r$ выше, чем для Э. При этом для всех исследованных мицеллярных систем с различными $R_{\mathrm{h}}$ степень анизотропии выше значений, полученных в водных растворах. Значения степени анизотропии флуоресценции в воде следующие: $r_{\ni}=0.04, r_{\ni р}=0.04, r_{\text {БР }}=0.05$. Это указывает на то, что движение молекул исследованных красителей имеет в обратной мицелле больше ограничений, чем в чистой воде. Кроме того, большее значение $r$ для малых $R_{\mathrm{h}}$ показывает, что микросреда внутри водного пула обратной мицеллы сильно отличается от объемной воды. При этом высокая вязкость наблюдается благодаря молекулам воды, которые прочно связаны с сульфонатной головной группой АОТ. Уменьшение степени анизотропии излучения с увеличением $R_{\mathrm{h}}$ (рис. 6) указывает на то, что молекулы красителей имеют больше свободного движения, которое достигается изменением расположения молекул красителей внутри мицеллы - они удаляются от головной группы сурфактанта к связанной воде в обратной мицелле.

Для установления расположения молекул красителей внутри мицелл оценим вращательную диффузию молекул красителя в зависимости от $R_{\mathrm{h}}$. Определим параметры вращательной диффузии люминесцирующих молекул с помощью формулы Перрена [27]:

$$
\frac{1}{r}=\frac{1}{r_{0}}\left(1+\frac{\langle\tau\rangle}{\theta}\right),
$$

где $\theta=\frac{\eta V}{k T}-$ время вращательной релаксации, $T-$ абсолютная температура, $\eta-$ вязкость раствора, $V$ объем молекул флуорофора, $k$ - постоянная Больцмана, $\langle\tau\rangle$ - среднее время жизни возбужденных молекул, $r_{0}$ - предельная анизотропия излучения. Уравнение (8) содержит линейную зависимость $\frac{1}{r}$ от $\frac{T}{\eta}$, поэтому изменяя вязкость раствора и измеряя степень анизотропии, можно получить значение $r_{0}$. Для определения $r_{0}$ мы провели измерения $r$ при различных значениях вязкости водных растворов, которая изменялась путем добавления сахарозы в растворы. Для каждого из растворов методом наименьших квадратов были построены зависимости $\frac{1}{r}\left(\frac{T}{\eta}\right)$ и получены значения $r_{0}$. Были получены следующие значения $r_{0}$ : Э-0.34, ЭР -0.27, БР 0.32 .

Используя измеренные значения $r, r_{0}$ и $\langle\tau\rangle$, для каждого из растворов с помощью формулы (8) было вычислено время вращательной релаксации $\theta$. На рис. 7

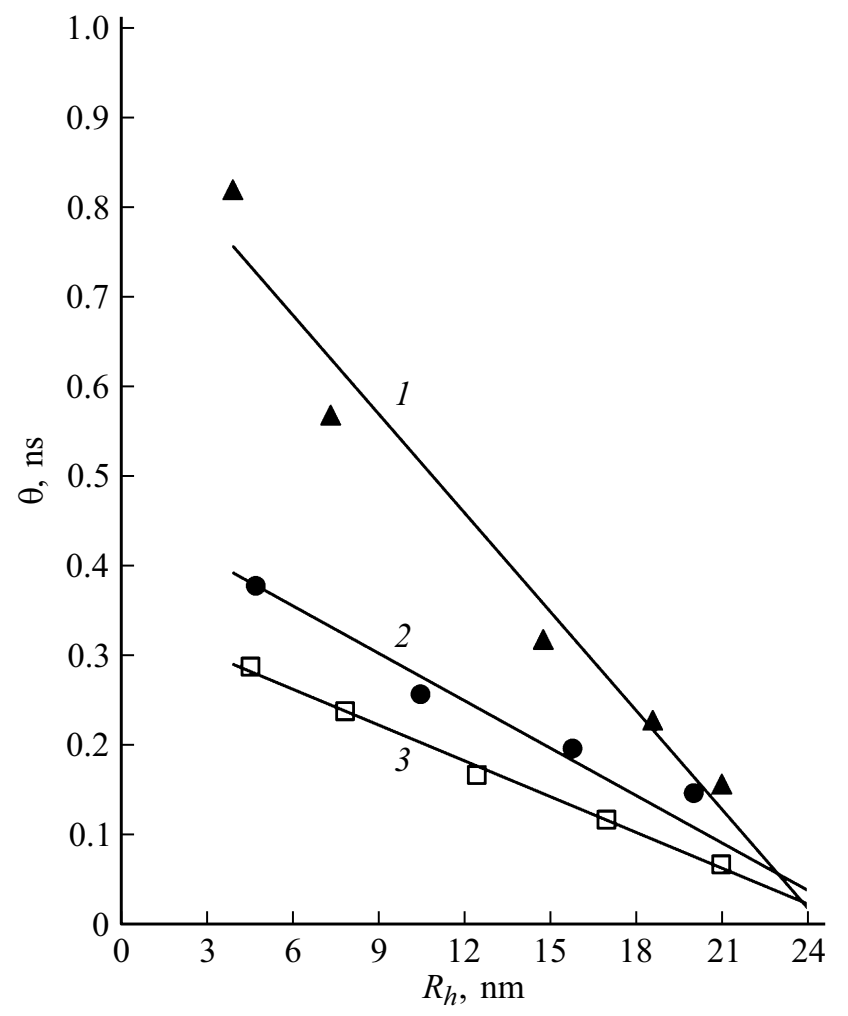

Рис. 7. Зависимости времени вращательной релаксации от гидродинамического радиуса $R_{\mathrm{h}}$ для Э (1), ЭР (2) и БР (3).

представлены зависимости времени вращательной релаксации $\theta$ от $R_{\mathrm{h}}$ для Э (кривая 1 ), ЭР (кривая 2), БР (кривая 3). Из рис. 7 видно, что $\theta_{\mathrm{E}}>\theta_{\mathrm{ER}}>\theta_{\mathrm{BR}}$, т.е. на значении времени вращательной корреляции сказывается „эффект тяжелого атома“. С ростом $R_{\mathrm{h}}$ происходит уменьшение $\theta$ для всех трех исследованных красителей. При этом „скорость“ уменьшения $\theta$ от увеличения размера (радиуса) мицеллы $d \theta=\frac{d \theta}{d R_{\mathrm{h}}}$ имеет следующие значения: $(d \theta)_{\mathrm{E}}=0.039,(d \theta)_{\mathrm{ER}}=0.015$ и $(d \theta)_{\mathrm{BR}}=0.013$. В связи с тем, что $\theta$ пропорционально вязкости $\eta$, наблюдаемое в эксперименте уменьшение $\theta$ с ростом гидродинамического радиуса мицеллы связано с уменьшением микровязкости ограниченной водной среды внутри мицеллы. Это подтверждает, что молекулы красителей удаляются от гидратированных полярных групп ПАВ в мицелле.

\section{Заключение}

Исследованы молекулы галогенпроизводных флуоресцеина в обратных мицеллах АОТ методом динамического рассеяния света, стационарной и времяразрешенной флуоресцентной спектроскопии. Установлено, что внедрение молекул красителей в обратные мицеллы вызывает увеличение их гидродинамических радиусов $R_{\mathrm{h}}$.

Обнаружено уменьшение среднего времени жизни $\langle\tau\rangle$ возбужденных состояний молекул исследованных 
красителей с ростом $R_{\mathrm{h}}$, что объясняется ростом подвижности молекул воды и ослаблением эффекта их геометрического ограничения в обратных мицеллах.

Анизотропия флуоресценции молекул красителей уменьшается с ростом $R_{\mathrm{h}}$, при этом для всех $R_{\mathrm{h}}$ степень анизотропии выше значений, полученных в водных растворах.

По анизотропии флуоресценции молекул красителей определено время вращательной релаксации $\theta$. Установлено, что $\theta_{\mathrm{E}}>\theta_{\mathrm{ER}}>\theta_{\mathrm{BR}}$, т.е. на значении времени вращательной релаксации сказывается „эффект тяжелого атома“. С ростом $R_{\mathrm{h}}$ происходит уменьшение $\theta$ для всех трех исследованных красителей, что связано с увеличением микровязкости ограниченной водной среды внутри мицеллы.

\section{Финансирование работы}

Исследование выполнено при финансовой поддержке РФФИ в рамках научного проекта № 19-32-90123.

\section{Конфликт интересов}

Авторы заявляют, что у них нет конфликта интересов.

\section{Список литературы}

[1] Быков А.В., Старокуров Ю.В., Салецкий А.М. // Опт. и спектр. 2020. Т. 128. С. 118. doi 10.21883/OS.2020.01.48847.247; Bykov A.V., Starokurov Yu.V., Saletsky A.M. // Opt. Spectrosc. 2020. V. 128. P. 114. doi 10.1134/S0030400X20010063

[2] Afanasyev D.A., Ibrayev N.Kh., Saletsky A.M. et al. // J. Luminesc. 2013. V. 136. P. 358. doi 10.1016/j.jlumin.2012.11.013

[3] Старокуров Ю.В., Летута С.Н., Пашкевич С.Н., Антропова Т.В., Гордеева Ю.А., Салецкий А.М. // Опт. и спектр. 2013. T. 114. № 1. C. 95. doi 10.7868/S003040341301025X; Starokurov Y.V., Gordeeva Y.A., Saletsky A.M., Letuta S.N., Pashkevich S.N., Antropova T.V. // Opt. Spectrosc. 2013. V. 114. N 1. P. 87. doi 10.1134/S0030400X13010256

[4] Alvarado Y., Muro C., Illescas J., Díaz María del Carmen, Riera F. // Biomolecul. 2019. V. 9. P. 164. doi 10.3390/biom 9050164

[5] Hashimoto T., Ye Y., Ui M, Ogawa T., Matsui T., Tanaka Y. // Biochem. Biophys. Res. Commun. 2019. V. 514. P. 31. doi 10.1016/j.bbrc.2019.04.062

[6] Lone I.H., Radwan N.R.E., Aslam J., Akhter A. // Current Nanosci. 2019. V. 15. P. 129. doi 10.2174/1573413714666180611075115

[7] Orellano M.S., Porporatto C., Silber J.J., Falcone R.D., Correa N.M. // Carbohydr. Polym. 2017. V. 171. P. 85. doi 10.1016/j.carbpol.2017.04.074

[8] Piazzini V., D’Ambrosio M., Luceri C., Cinci L., Landucci E., Bilia A.R., Bergonzi M.C. // Molecules. 2019. V. 24. P. 1688. doi 10.3390/molecules24091688

[9] Singh P., Verma N. // Int. J. Pharm. Sci. Res. 2018. V. 9. P. 1397. doi 10.13040/IJPSR.0975-8232.9(4).1397-04
[10] Rahdar A., Almasi-Kashi M., Khan A.M., Aliahmad M., Salimi A., Guettari M., Kohne H.E.G. // J. Molec. Liq. 2018. V. 252. P. 506. doi 10.1016/j.molliq.2018.01.004

[11] Rahdar A., Aliahmad M., Kor A.M., Sahoo D. // Spectrochim. Acta A. 2019. V. 210. P. 165. doi 10.1016/j.saa.2018.11.015

[12] Bozkurt E., Onganer Ya. // J. Molec. Struct. 2018. V. 1173. P. 490. doi 10.1016/j.molstruc.2018.07.019

[13] Hoseini M., Sazgarnia A., Sharifi S. // Opt. Quant. Electron. 2019. V.51. P.144. doi 10.1007/s11082-019-1865-1

[14] Rahdar A., Salmani S., Sahoo D. // J. Molec. Struct. 2019. V. 1191. P. 237. doi 10.1016/j.molstruc.2019.04.083

[15] Peyghami S., Sharifi S., Rakhshanizadeh F., Alizadeh Kh. // J. Molec. Liq. 2017. V. 246. P. 157. doi 10.1016/j.molliq.2017.09.058

[16] Karimi N., Sharifi S., Parhizgar S.S., Elahi S.M. // Opt. Quant. Electron. 2018. V. 50. P. 209. doi 10.1007/s11082018-1478-0

[17] Волкова О.И., Баранов А.Н., Салецкий А.М. // ЖПС. 2018. T. 85. № 3. С. 373; Volkova O.I., Baranov A.N., Saletsky A.M. // J. Appl. Spectr. 2018. V. 85. N 3. P. 381. doi 10.1007/s10812-018-0661-1

[18] Dhillon S.K., Porter S.L., Rizk N., Sheng Y., McKaig Th., Burnett K., White B., Nesbitt H., Matin R.N., McHale A.P., Callan B., Callan J.F. // J. Med. Chem. 2020. V. 63. P. 1328. doi 10.1021/acs.jmedchem.9b01802?ref=pdf

[19] Naranjo A., Arboleda A., Martinez J.D., Durkee H., Aguilar C., Relhan N., Nikpoor N., Galor A., Dubovy S.R., Leblanc R., Flynn H.W., Miller D., Parel J.-M., Amescua G. // Amer. J. Ophthalmol. 2019. V. 208. P. 387. doi 10.1016/j.ajo.2019.08.027

[20] Shitomi K., Miyaji H., Miyata S., Sugaya T., Ushijima N., Akasaka T., Kawasaki H. // Photodiag. Photodyn. Therapy. 2020. V. 30. P. 101647. doi 10.1016/j.pdpdt.2019.101647

[21] Летута С.Н., Пашкевич С.Н., Ииемгулов А.Т., Никиян А.Н. // Биофизика. 2020. Т. 65. № 4. С. 705-712. doi 10.31857/S0006302920040109

[22] Vlasova I.M., Saletsky A.M. // Las. Phys. 2010. V. 20. P. 1844. doi 10.1134/S1054660X10170160

[23] Vlasova I.M., Saletsky A.M. // J. Molec. Struct. 2009. V. 936. P. 220. doi 10.1016/j.molstruc.2009.07.043

[24] Городничев Е.С., Кулешова А.А., Быков А.В., Салецкий A.M. // ЖПС. 2019. Т. 86. № 5. С. 773; Gorodnichev E.S., Kuleshova A.A., Bykov A.V., Saletsky A.M. // J. Appl. Spectr. 2019. V. 86. P. 855. doi 10.1007/s10812-019-00906-1

[25] Potapov A.V., Alekseev D.B., Alekseeva I.G., Saletsky A.M. // Las. Phys. Lett. 2007. V. 4. P. 61. doi 10.1002/lapl.200610060

[26] lonis N., Sawyer W.H. // J. Fluoresc. 1996. V. 6. N 3. P. 147. doi 10.1007/BF00732054

[27] Левшин Л.В., Салецкий А.М. Люминесценция и ее измерения. Молекулярная люминесценция. М.: Изд-во МГУ, 1989. $272 \mathrm{c}$. 